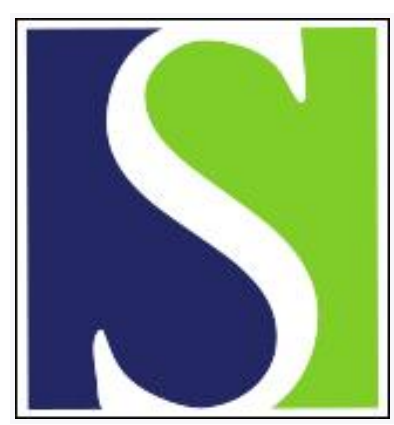

Scand J Work Environ Health 2008;34(6):407-409

https://doi.org/10.5271/sjweh.1294

Issue date: 00 Dec 2008

Role of occupational hygiene research in the control of occupational health risks from engineered nanoparticles

by Schneider T

Affiliation: Scandinavian Journal of Work, Environment \& Health, Topeliuksenkatu 41 a A, FI-00250 Helsinki. ts@ami.dk

Refers to the following texts of the Journal: 2001;27(5):354-357 2008;34(6):471-478

Key terms: control; editorial; engineered nanoparticles; health risk; nanoparticle; occupational health risk; occupational hygiene research

This article in PubMed: www.ncbi.nlm.nih.gov/pubmed/19137201

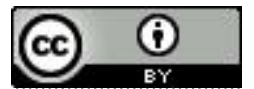




\section{Role of occupational hygiene research in the control of occupational health risks from engineered nanoparticles}

In the 1980s, there was a growing awareness that the approaches to the occupational exposure assessment of air contaminants used at the time may not have been sufficiently sophisticated for the complex exposure scenarios that had emerged. With the purpose of initiating the development of new paradigms for assessing inhalation exposure, a workshop on exposure assessment for epidemiology and hazard control was organized at Woods Hole, Massachusetts, in 1988. In order to illustrate the challenges to be addressed, the editors of the workshop proceedings rephrased the dilemma of science ". . . you can only see what you look for, and what you see depends on how you look ..." as ". . o our formulation of hypotheses (what we look for) limits the interpretation of observations (what we can see), while the resulting choice of methods (how we look) restricts the observations we can make" (1). Among the concerns were that highly reactive toxic compounds may have disappeared from the sample before analysis, that unnecessary analytical efforts using ever more powerful analytical tools could provide much data that could be of limited relevance, and that a limited number of measurements would not describe complex exposure scenarios. These concerns illustrated the ". . . conflict between the simple desire to measure and the inherent lack of information concerning toxicological factors and exposure variability ..." (1). Similar challenges, concerns, and conflicts have re-emerged in the case of engineered nanoparticles due to the rapid development of their production and use. In this issue of the Scandinavian Journal of Work, Environment \& Health, Schulte et al (2) have focused on seven critical questions that need to be addressed in order to explore the safety of engineered nanoparticles more effectively. They stress the need for extensive exposure assessment and characterization in order to answer one of their critical questions "What is the exposure to engineered nanoparticles in the workplace?" The lack of exposure data is, in part, caused by the lack of a consensus about which particle characteristics or exposure metrics should be used-another key question posed by Schulte et al (2).

Nanoparticles follow the same aerodynamic laws as other particles. Thus the exposure determinants that influence the transport from source to receptor (worker) are the same for both engineered nanoparticles and other particles, and the assessment of their exposure can build on the vast experience gained from the exposure assessment of classical aerosols (2), including exposure modeling approaches. As small, lightweight, battery-driven pumps and analytical methods with ever lower detection limits became available, it became possible to make extensive measurements of air contaminants in the breathing zone of individual workers. A major message at the Woods Hole workshop was that these measurements had shown that the day-to-day exposure variability for a given worker and between workers doing the same job group could be very large and that within-worker variance was often larger than between-worker variance. Since then, considerable progress in the statistical modeling of exposure variability has been made, enabled by the increase in the computing power of personal computers and the availability of userfriendly software. This work has led to the development of measurement strategies and statistical models that provide exposure information that can be used for multiple purposes, including hazard evaluation, control, and epidemiology (3).

Roach (4) was probably the first to use mechanistic modeling for retrospective exposure assessment in epidemiology. He used the quantifiable exposure determinants engineering control (ventilation) and production rate (average length of colliery face worked) to estimate previous dust exposures in collieries. While mechanistic modeling has been applied extensively for ambient air pollution, mechanistic 
occupational exposure modeling has, until recently, received much less attention. The need for developing and, in particular, validating mechanistic exposure models is now apparent due to the new European chemicals regulation REACH (registration, evaluation, authorization, and restriction of chemical substances), which requires occupational exposure assessment (5) for a potentially huge number of specific exposure scenarios.

A consensus report (6) based on the conference "X2001 Exposure Assessment in Epidemiology and Practice" recommends that one should always broaden the context within which the initial problem is framed, and, in its view, exposure determinants should conceptually be divided into micro(individual)-, meso(workplace)-, and macro(societal)-level factors. These ideas have received limited attention in relation to exposure to air contaminants. Within-group exposure variability is often substantial, even when a large set of workplace factors is used in statistical models, and the same would also be expected for engineered nanoparticles. Rather than treating this variability as residual variance, one could investigate whether or not small residual variances were positively associated with meso-level factors such as the high quality of on-the-job training or safety culture. If so, it would document the significance of these factors in protecting the health of all workers. In addition, macro-level factors, such as labor resources or competitive pressures, could potentially influence exposure to engineered nanoparticles.

Exposure to air contaminants can be assessed with different methods, such as expert assessment, modeling, and (an often limited number of) measurements. One line of research has been to develop approaches for combining these methods into one comprehensive strategy using a Bayesian framework. Tielemans et al (7) used this approach in their higher tier, exposure assessment tool for REACH. The initial assessment (or prior belief in Bayesian terminology) of exposure for a given scenario is calculated using a mechanistic model, and the result is combined (updated in Bayesian terminology) with empirical data from an exposure database. In the updating, an algorithm based on the mechanistic model accounts for the degree of similarity between the scenario to be assessed and the scenarios for which measurement data are available. In this approach, optimal use is made of available empirical exposure data. Bayesian updating may be an unfamiliar method, despite the fact that scientists use this method all the time. Scientists must reason on the basis of uncertain propositions, and decide how they should change how much they believe in a hypothesis, given the new information that has been collected. It was shown by the American physicist Richard Cox in 1946 that the way to proceed logically and consistently is via a rule that turns out to be Bayes' theorem (8).

Schulte et al (2) recommend that an adhoc database be established to promote faster dissemination and to focus attention on methods currently being used. To maximize the benefit, much attention should be given to the contextual information for characterizing exposure modifiers that should be collected and stored. Statistical model parameters (workplace factors) are often chosen on a case-by-case basis, and the approach has made it difficult to combine the knowledge from different studies in order to identify the modifiers and the magnitude of their effects on exposure. Thus a set of uniquely identifiable and quantifiable exposure modifiers should be developed prior to measurements being carried out. Such a list would have implications for the design of measurement strategies and for the data analysis, and it will facilitate the exchange of data. Preferably, the choice of modifiers should consider the progress made in mechanistic modeling, in which exposure modifiers have been based on first principles and thus are generally applicable. Such an effort would enhance the usability of measurement data and facilitate the further development and validation of mechanistic models.

Once during my early days as an occupational hygienist, I was doing field measurements in a metal foundry. I became fascinated by the large white "snow flakes" that suddenly started settling through the air. I was given the explanation that they were large agglomerates of nano-sized zinc oxide particles that had coagulated rapidly due to the high concentration and small size of primary zinc fume particles. I could not have foreseen that the coagulation of engineered nanoparticles would become a major issue 
more than 30 years later. If, for example, engineered nanoparticles from a production process escape into the workroom air, their concentration will often be much higher than the background concentration of particles, and they will coagulate rapidly and form agglomerates and thus are present in particles of larger size than the primary particles. If the concentration of engineered nanoparticles is much lower than the background levels, they will rapidly become attached to larger background particles. This attachment makes them "invisible" if instruments that only measure particle size are used (9). Maybe seeing them is not absolutely necessary, provided one characterizes the engineered nanoparticles at the source, as well as the background particles. Because the agglomeration process is reasonably well understood, one could attempt to develop simple models for estimating the fraction of the overall exposure to engineered nanoparticles constituted by those that end up attached to background particles. The degree to which agglomerated or attached nanoparticles de-agglomerate in lung fluids and present their nanoproperties is another important characteristic. Innovative methods for quantifying this characteristic will be needed.

Occupational hygiene researchers who are approaching retirement (and I belong to this group) can be pleased that their field of research, to which they devoted their professional life, has contributed to the significant reduction in worker health risk from exposure to air contaminants. They can also feel contented that this research has established a solid foundation for addressing the challenges identified by Schulte et al (2). It is of concern, however, that the demand for occupational hygiene expertise has declined and that, in the future, much of the accumulated knowledge may not be fully exploited or may be overlooked. The emergence of nanotechnology has demonstrated the importance of occupational hygiene and presents challenges. These challenges will hopefully attract young researchers.

\section{References}

1. Smith TJ, Rappaport SM. Introduction. In: Rappaport SM, Smith TJ, editors. Exposure assessment for epidemiology and hazard control. Chelsea (MI): Lewis Publishers Inc;1991. p ix-xiv.

2. Schulte P, Geraci C, Zumwalde R, Hoover M, Castranova V, Kuempel E, et al. Sharpening the focus on occupational safety and health of nanotechnology. Scand J Work Environ Health. 2008;34(6):471-78.

3. Kromhout H. Design of measurement strategies for workplace exposures. Occup Environ Med. 2002;59:349-54.

4. Roach SA. A method of relating the incidence of pneumoconiosis to airborne dust exposure. Br J Ind Med. 1953;10:2206.

5. European Chemicals Agency (ECHA). Guidance on information requirements and chemical safety assessment, chapter R.14: occupational exposure estimation [Internet]. Helsinki: ECHA; 2008 [Cited: 7 November 2008]. Available from: http://reach.jrc.it/docs/guidance_document/information_requirements_r14_en.pdf?vers=20_08_08.

6. Broadening the view of exposure assessment [consensus report]. Scand J Work Environ Health. 2001;27(5):354-57.

7. Tielemans E, Warren N, Schneider T, Tischer M, Ritchie P, Goede H, et al. Tools for regulatory assessment of occupational exposure: development and challenges. J Expo Sci Environ Epidemiol. 2007;17:S72-S80.

8. Matthews R. Base instincts. New Sci [Internet]. 2000;29 January [Cited: 25 November 2009]. Available from: http://www. newscientist.com/article/mg16522234.900-base-instincts.html.

9. Seipenbusch M, Binder A, Kasper G. Temporal evolution of nanoparticle aerosol in workplace exposure. Ann Occup Hyg. 2008:52:707-16.

Thomas Schneider, Associate Editor

Scandinavian Journal of Work Environment \& Health

Helsinki, Finland

[ts@nrcwe.dk] 
